\title{
The clinical efficacy of L-DOPA and STN-DBS share a common marker: reduced GABA content in the motor thalamus
}

\author{
A Stefani ${ }^{1,2}$, E Fedele $^{3,4}$, J Vitek $^{5}$, M Pierantozzi ${ }^{1,2}$, S Galati ${ }^{1,2}$, F Marzetti $^{2}$, A Peppe $^{1}$, MS Bassi ${ }^{2}$, G Bernardi $^{1,2}$ and P Stanzione ${ }^{\star, 1,2}$
}

At odd with traditional views, effective sub-thalamic nucleus (STN) deep brain stimulation (DBS), in Parkinson's disease (PD) patients, may increase the discharge rate of the substantia nigra pars reticulata and the internal globus pallidus (GPi), in combination with increased cyclic guanosine monophosphate (cGMP) levels. How these changes affect the basal ganglia (BG) output to the motor thalamus, the crucial structure conveying motor information to cortex, is critical. Here, we determined the extracellular GABA concentration in the ventral anterior nucleus (VA) during the first delivery of STN-DBS $(n=10)$ or following levodopa $(L D)(n=8)$. Both DBS and subdyskinetic LD reversibly reduced $(-30 \%)$ VA GABA levels. A significant correlation occurred between clinical score and GABA concentration. By contrast, only STN-DBS increased GPi cGMP levels. Hence, STN$\mathrm{ON}$ and MED-ON involve partially different action mechanisms but share a common target in the VA. These findings suggest that the standard BG circuitry, in PD, needs revision as relief from akinesia may take place, during DBS, even in absence of reduced GPi excitability. However, clinical amelioration requires fast change of thalamic GABA, confirming, in line with the old model, that VA is the core player in determining thalamo-cortical transmission.

Cell Death and Disease (2011) 2, e154; doi:10.1038/cddis.2011.35; published online 5 May 2011

Subject Category: Neuroscience

The classical basal ganglia (BG) model $^{1,2}$ postulated that, in Parkinson's disease (PD) akinesia results from a functional imbalance between indirect and direct pathways. An overactivity of the sub-thalamic nucleus (STN) and the internal segment of the globus pallidus (GPi) was supposed to have a key role in determining the OFF state. As similar benefits were obtained by STN deep brain stimulation (DBS) or STN lesion, it was suggested that STN-DBS represented a sort of functional ablation ('the suppression hypothesis', see Filali et $a l^{3}$ ).

Some experimental studies using the 6-hydroxydopamine (6-OHDA) rodent's model corroborated those assumptions (Benazzouz et al. ${ }^{4}$; Windels et al., ${ }^{5,6}$ but consider Shi et al. ${ }^{7}$ ).

Alternatively, the efficacious STN-DBS, when delivered in PD patients and non-human primates, increased-and not decreased-GPi and substantia nigra pars reticulata $(\mathrm{SNr})$ discharge rate while modulating their pattern of activity; during STN-DBS GPi neuronal activity was indeed more regular, timelocked to the stimulus pulse, and had less bursting activity. ${ }^{8-10}$ When inhibition was found, ${ }^{11}$ it derived from short microstimulation periods, presumably confined to the cell bodies.

Moreover, first by disease modeling, ${ }^{12}$ and later by extracellular recordings during STN-DBS in 1-methyl-4phenyl-1,2,3,6-tetrahydropyridine (MPTP)-intoxicated primates, those unexpected changes in GPi were found to be associated with a modification in the pattern of neuronal activity in the pallidal (ventralis anterior, ventral anterior nucleus (VA), as in the terminology of Hassler et $a{ }^{13}{ }^{13}$ ) and cerebellar $(\mathrm{VL})$ receiving areas of the motor thalamus. The resulting neuronal activity in both the thalamic regions became increasingly periodic and regular, with a shift in oscillatory activity from low to high frequencies. ${ }^{14}$ Therefore, all these data indicate that some premises of the standard model, if centered on the putative hyperactivity of specific 'prethalamic' pathways, should be wrong, whereas not enough attention was, so far, dedicated to the biochemical counterpart of those electrophysiological evidence.

Here, our aim was to identify any clinically related biochemical change in GABA content occurring in the VA. In addition, we asked whether different acute treatments, namely levodopa (LD) or DBS, causing similar therapeutic effects, also share biochemical parameters in the VA as a possible 'common transducer of efficacy'.

In support of a common pathway, the maximal clinical improvement by STN-DBS in the early postoperative stage usually does not exceed that obtained with LD. ${ }^{15,16}$ There is also evidence against a common pathway; for example, the different impact on OFF dystonia. Further, LD-mediated

\footnotetext{
${ }^{1}$ Neurofisiopatologia, IRCCS Fondazione S Lucia, Roma, Italy; ${ }^{2}$ Clinica Neurologica, Dipartimento di Neuroscienze, Università di Roma Tor Vergata, Roma, Italy; ${ }^{3}$ Dipartimento di Medicina Sperimentale, Sezione di Farmacologia e Tossicologia, Università degli Studi di Genova, Genova, Italy; ${ }^{4}$ Centro di Eccellenza per la Ricerca Biomedica, Università degli Studi di Genova, Genova, Italy and ${ }^{5}$ Department of Neurology, University of Minnesota, Minneapolis, MN, USA

*Corresponding author: P Stanzione, Neurofisiopatologia, IRCCS Fondazione S Lucia, Via Ardeatina 306, Roma 00133, Italy. Tel: + 390620903115 ;

Fax: + 39062090 3118; E-mail: stanzione@ @med.uniroma2.it

Keywords: Parkinson's disease; STN-DBS; challenge test; pallido-thalamic pathway

Abbreviations: STN, sub-thalamic nucleus; DBS, deep brain stimulation; PD, Parkinson's disease; GPi, internal globus pallidus; cGMP, cyclic guanosine monophosphate; VA, ventral anterior nucleus; LD, levodopa; BG, basal ganglia; OHDA, 6-hydroxydopamine; SNr, substantia nigra pars reticulata; MPTP, 1-methyl-4phenyl-1,2,3,6-tetrahydropyridine; UPDRS, Unified Parkinson's Disease Rating scale; CP, caudate-putamen

Received 07.1.11; revised 23.2.11; accepted 08.3.11; Edited by G Melino
} 
and DBS-mediated effects on limbic and associative functions may differ strongly, ${ }^{17,18}$ suggesting that incomplete mechanisms overlap.

Here, we took advantage of the 'multi-target DBS strategy', where several PD patients were included to the bilateral implantation of both STN and GPi; ${ }^{16,19-21}$ this allowed to collect - and correlate with clinical scores - microdialysis data from the GPi and VA of the same patients (Figure 1). In particular, we studied whether clinically effective STN-DBS $(185 \mathrm{~Hz})$ or intra-operative LD administration (at a subdyskinetic dose) influenced GABA levels in the VA. In addition, as our previous investigations had shown that STN-DBS induced a large increase of cyclic guanosine monophosphate (cGMP) concentration in the GPi, we evaluated to what extent LD did replicate that finding, which in turn would establish whether the nucleotide alteration is a critical, and clinically related, feature, shared by both therapeutic approaches.

The present work did not explore all the putative targets of STN-DBS and LD; however, our data emphasize that, while there may be some differences between LD and DBS mechanisms, as indicated by the DBS-mediated increase of GPi cGMP (in contrast with LD), the reduced GABA availability inside the VA, seen with both LD and DBS, may represent one critical index of any effective therapy for $P D$ rigidity and akinesia.

\section{Results}

\section{Effects of STN-DBS}

Thalamic GABA changes. DBS produced a significant decrease in GABA content during stimulation (Table 1 and Figure 2; average 31.1\%; ANOVA main effect $\mathrm{df}=2 / 18$; $\varepsilon=0.784, \mathrm{df}=1.56 / 14.11 ; \mathrm{F}=11.83, P=0.000523 ; 0.0016$ Greenhause-Geisser correction). Post hoc Sheffé test showed the following: basal versus DBS $P=0.000783$; DBS versus recovery $P=0.00413$. Friedman ANOVA followed by Wilcoxon confirmed these levels of significance. In two cases, DBS was kept at $0 \mathrm{~V}$ for $20 \mathrm{~min}$ and no consistent change of GABA was detected (not shown).

Pallidal CGMP changes. DBS produced a clear-cut increase of cGMP as already described (Table 1 and Figure 2; average 606.2\%; ANOVA main effect $\mathrm{df}=2 / 18 ; \varepsilon=0.515$, $\mathrm{df}=1.03 / 9.28 ; \mathrm{F}=20.56, \quad P=0.00002)$. Post hoc Sheffé test showed: basal versus DBS $P=0.000047$; DBS versus recovery $P=0.000489$. Friedman ANOVA followed by Wilcoxon test confirmed these significances.

Clinical effect of DBS. There was a significant change of Unified Parkinson's Disease Rating scale (UPDRS) score during STN-DBS (Table 1 and Figure 1; $n=10 ; \mathrm{df}=17$; $\chi^{2}=143.12 ; \quad P=0.000001$ Friedman ANOVA). Post hoc Wilcoxon test showed a significant UPDRS score amelioration from the first $10 \mathrm{~min}$ of DBS in comparison with all the basal scores. The score was significantly lower throughout DBS and during the first $10 \mathrm{~min}$ of recovery.

Correlations. Clinical score significantly correlated with cGMP increase ( $R$ Spearman: $-0.73, P<0.05$ ) and GABA

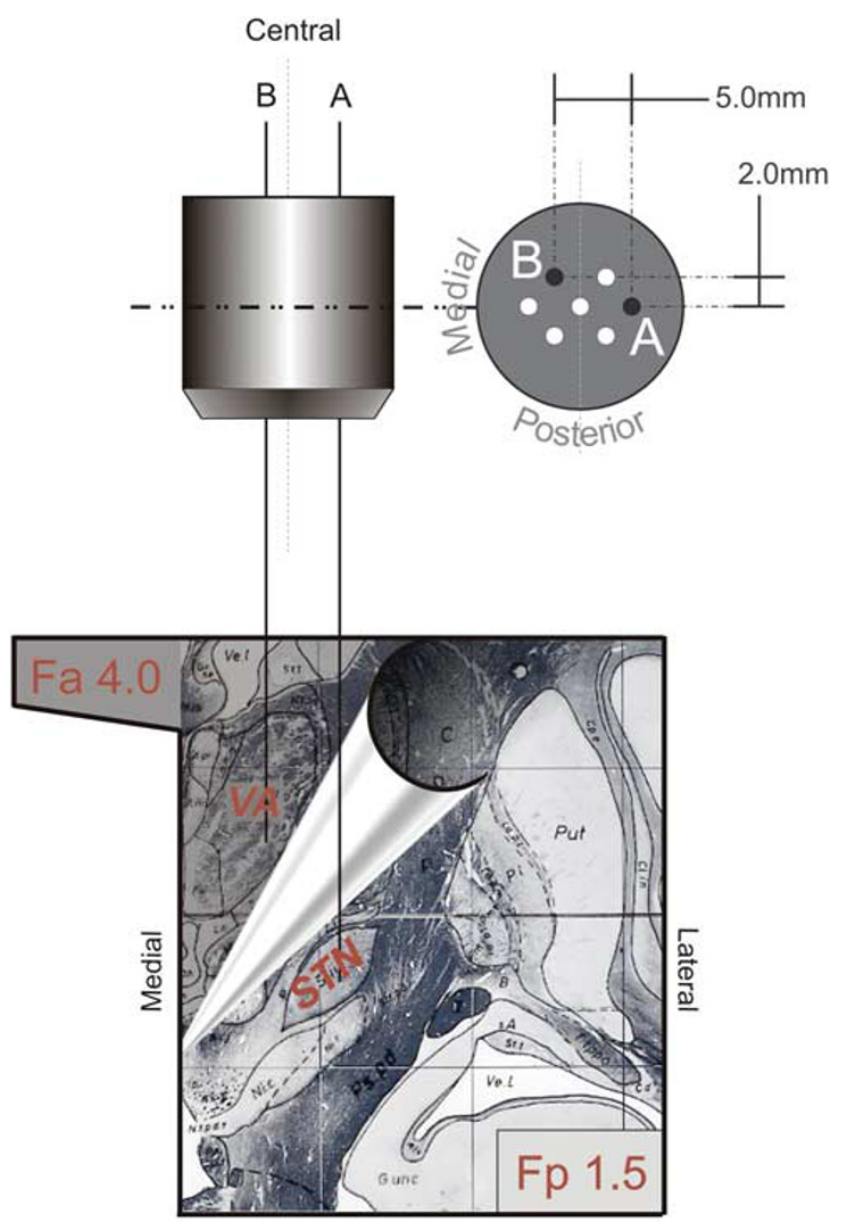

Figure 1 Trajectories of the multi-electrode probes holders aimed at the STN-VA. Each section shows representative diagrams of the multi-electrode holder (top) used and, at the bottom, two different partially superimposed sections of atlas (note the hand leafing the superior section). The bottom panel shows a coronal atlas section at $1.5 \mathrm{~mm}$ posterior to the inter-commissural midline (Fp 1.5, STN target) and at $4 \mathrm{~mm}$ anterior to the inter-commissural midline ( $\mathrm{Fa} 4.0 \mathrm{VA}$ target). To reach the STN passing through the VA, the central electrode of the multi-electrode holder (upper part) was aimed at a target at $8.5 \mathrm{~mm}$ of laterality, $-4 \mathrm{~mm}$ with respect to the AC-PC plane, and at the mid-point of the AC-PC line. The trajectory had an angle of $70^{\circ}-75^{\circ}$ in the sagittal plane and of $85^{\circ}-90^{\circ}$ in the coronal plane. The more lateral electrode of the multi-electrode holder was at $3.5 \mathrm{~mm}$ of laterality from the central one. This laterality allowed to reach a target at $12.0 \mathrm{~mm}$ of laterality from the midline, which is the usual location of the STN target. On the contrary, the trajectory of electrode-B was $1.5 \mathrm{~mm}$ more medial than the central one and $2 \mathrm{~mm}$ more anterior. The probe membrane ( $4 \mathrm{~mm}$ length) was positioned between 11.0 and $7.0 \mathrm{~mm}$ above the target $(+7$ to $+3 \mathrm{~mm}$ above the inter-commissural plane), along the trajectory of electrode- $\mathrm{B}$. Given the angle in the sagittal planes $\left(70^{\circ}-75^{\circ}\right)$, the trajectory had a laterality of $7.0 \mathrm{~mm}$ and, at the level of +7 to $+3 \mathrm{~mm}$ above the inter-commissural plane, its anterior-posterior position was between +6 and $+4 \mathrm{~mm}$ with respect to the midpoint of the inter-commissural line, corresponding to the center of the VA

decrease (R: $+0.67, P<0.05)$. No significant correlation was observed between GABA and cGMP changes.

\section{LD Effects}

Thalamic GABA changes. LD produced a significant decrease of GABA content during $2 \mathrm{~h}$ after LD administration (Table 2 and Figure 3; ANOVA main effect $\mathrm{df}=2 / 10$; $\varepsilon=0.687, \mathrm{df}=1.37 / 6.87 ; \mathrm{F}=35.57, P=0.000028 ; 0.000387$ 
Table 1 Microdialysis values and clinical scores before, during, and after STN-DBS

\begin{tabular}{|c|c|c|c|c|c|c|c|c|c|c|c|c|c|c|c|c|c|c|c|c|c|}
\hline \multirow[b]{3}{*}{ Patient } & \multicolumn{7}{|c|}{ Basal } & \multicolumn{7}{|c|}{ STN-DBS } & \multicolumn{7}{|c|}{ Recovery } \\
\hline & \multicolumn{20}{|c|}{ cGMP-GPi } & \multirow[b]{2}{*}{ Mean } \\
\hline & & & & & & & Mean & & & & & & & Mean & & & & & & & \\
\hline 1 & 2.4 & 2.2 & 1.9 & 2.4 & 2.1 & 2.2 & 2.2 & 4.4 & 9.7 & 24.5 & 22.1 & 29.7 & 30.1 & 20.1 & 28.5 & 2.4 & 1.3 & 1.1 & 2.1 & 2.3 & 6.3 \\
\hline 2 & 1.7 & 1.3 & 1.7 & 1.5 & 1.4 & 1.5 & 1.5 & 2.7 & 2.3 & 2.5 & 3.2 & 3.1 & 3.4 & 2.9 & 3.9 & 2.1 & 1.5 & 1.4 & 1.2 & 1.3 & 1.9 \\
\hline 3 & 2.5 & 1.9 & 2.4 & 1.6 & 2.0 & 2.1 & 2.1 & 3.0 & 2.7 & 2.3 & 2.3 & 2.5 & 2.9 & 2.6 & 3.2 & 2.0 & 1.5 & 1.1 & 2.1 & 2.3 & 2.0 \\
\hline 4 & 1.0 & 1.5 & 1.1 & 1.0 & 1.0 & 1.1 & 1.1 & 2.5 & 9.4 & 10.7 & 12.6 & 10.9 & 11.2 & 9.6 & 12.1 & 1.9 & 1.1 & 1.4 & 1.1 & 1.4 & 3.2 \\
\hline 5 & 1.3 & 1.1 & 1.7 & 1.3 & 1.2 & 1.3 & 1.3 & 4.4 & 8.2 & 16.4 & 15.5 & 17.2 & 16.1 & 13.0 & 15.2 & 2.2 & 1.2 & 1.1 & 1.3 & 1.4 & 3.7 \\
\hline 6 & 1.9 & 2.1 & 2.8 & 2.3 & 2.4 & 2.3 & 2.3 & 3.1 & 2.8 & 3.5 & 5.9 & 6.4 & 5.1 & 4.5 & 13.4 & 2.7 & 1.8 & 1.5 & 2.2 & 1.5 & 3.9 \\
\hline $7^{\mathrm{a}}$ & 1.0 & 1.1 & 1.5 & 1.0 & 1.0 & 1.1 & 1.1 & 3.5 & 4.9 & 12.3 & 13.6 & 13.1 & 13.0 & 10.1 & 5.6 & 2.3 & 1.2 & 1.3 & 1.1 & 1.2 & 2.1 \\
\hline 8 & 1.3 & 1.0 & 1.3 & 1.2 & 1.4 & 1.2 & 1.2 & 1.4 & 3.7 & 10.0 & 10.6 & 9.2 & 11.0 & 7.7 & 4.1 & 2.0 & 1.4 & 1.2 & 1.3 & 1.1 & 1.9 \\
\hline 9 & 1.7 & 1.3 & 1.4 & 1.5 & 1.7 & 1.5 & 1.5 & 2.1 & 11.9 & 17.9 & 17.8 & 17.9 & 16.9 & 14.1 & 8.2 & 2.3 & 1.2 & 1.8 & 1.1 & 1.7 & 2.7 \\
\hline $10^{\mathrm{a}}$ & 1.7 & 1.5 & 1.7 & 1.8 & 1.7 & 1.7 & 1.7 & 4.4 & 8.2 & 14.2 & 15.5 & 15.3 & 16.1 & 12.3 & 13.1 & 3.2 & 1.2 & 1.5 & 1.3 & 1.4 & 3.6 \\
\hline Mean & 1.7 & 1.5 & 1.8 & 1.6 & 1.6 & 1.6 & 1.6 & 3.2 & 6.4 & 11.4 & 11.9 & 12.5 & 12.6 & 9.7 & 10.7 & 2.3 & 1.3 & 1.3 & 1.5 & 1.6 & 3.1 \\
\hline S.D. & 0.5 & 0.4 & 0.5 & 0.5 & 0.5 & 0.4 & 0.5 & 1.0 & 3.5 & 7.3 & 6.4 & 8.1 & 8.1 & 5.7 & 7.7 & 0.4 & 0.2 & 0.2 & 0.5 & 0.4 & 1.6 \\
\hline \multicolumn{22}{|c|}{ GABA-VA } \\
\hline 1 & 5.1 & 4.7 & 4.5 & 4.8 & 5.3 & 4.9 & 4.9 & 3.2 & 3.1 & 3.3 & 3.5 & 3.4 & 3.1 & 3.3 & 3.8 & 4.0 & 4.0 & 4.2 & 4.5 & 4.5 & 4.2 \\
\hline 2 & 4.0 & 3.9 & 3.7 & 4.3 & 4.1 & 4.0 & 4.0 & 3.0 & 4.0 & 3.5 & 3.9 & 3.2 & 3.0 & 3.4 & 3.0 & 3.3 & 4.1 & 5.4 & 6.3 & 5.0 & 4.5 \\
\hline 3 & 4.2 & 4.1 & 4.3 & 4.7 & 4.5 & 4.4 & 4.4 & 3.9 & 4.1 & 3.9 & 4.1 & 3.8 & 3.8 & 3.9 & 3.4 & 4.0 & 5.8 & 6.2 & 5.3 & 5.7 & 5.1 \\
\hline 4 & 4.3 & 4.0 & 4.5 & 4.9 & 4.3 & 4.4 & 4.4 & 3.9 & 3.7 & 4.0 & 4.0 & 3.8 & 3.9 & 3.9 & 3.7 & 3.7 & 3.9 & 3.5 & 3.4 & 3.7 & 3.7 \\
\hline 5 & 4.9 & 4.3 & 4.1 & 4.5 & 4.7 & 4.5 & 4.5 & 4.1 & 4.2 & 3.9 & 3.8 & 4.1 & 4.1 & 4.0 & 1.5 & 2.3 & 3.0 & 3.5 & 4.0 & 3.4 & 3.0 \\
\hline 6 & 5.1 & 5.6 & 5.4 & 5.3 & 5.5 & 5.4 & 5.4 & 3.5 & 2.1 & 2.0 & 2.0 & 2.1 & 1.7 & 2.2 & 5.0 & 5.0 & 5.5 & 5.3 & 6.3 & 6.0 & 5.5 \\
\hline $7^{\mathrm{a}}$ & 4.0 & 4.1 & 4.5 & 4.1 & 4.8 & 4.3 & 4.3 & 2.7 & 2.7 & 2.8 & 2.7 & 2.7 & 3.2 & 2.8 & 3.8 & 4.1 & 4.5 & 4.3 & 4.2 & 4.5 & 4.2 \\
\hline 8 & 4.9 & 5.1 & 5.3 & 4.9 & 4.7 & 5.0 & 5.0 & 2.9 & 2.6 & 2.4 & 2.3 & 2.3 & 2.3 & 2.5 & 3.5 & 3.2 & 4.1 & 4.5 & 4.8 & 5.0 & 4.2 \\
\hline 9 & 4.1 & 3.7 & 3.7 & 4.1 & 3.6 & 3.8 & 3.8 & 3.1 & 2.7 & 1.5 & 1.7 & 1.2 & 1.4 & 1.9 & 2.0 & 2.6 & 3.2 & 3.1 & 3.9 & 4.1 & 3.2 \\
\hline $10^{\mathrm{a}}$ & 4.5 & 4.5 & 4.0 & 4.0 & 4.4 & 4.3 & 4.3 & 3.9 & 3.0 & 2.6 & 2.4 & 2.4 & 2.4 & 2.8 & 4.7 & 5.0 & 5.1 & 5.3 & 4.9 & 4.7 & 5.0 \\
\hline Mean & 4.5 & 4.4 & 4.4 & 4.6 & 4.6 & 4.5 & 4.5 & 3.4 & 3.2 & 3.0 & 3.0 & 2.9 & 2.9 & 3.1 & 3.4 & 3.7 & 4.3 & 4.5 & 4.8 & 4.7 & 4.2 \\
\hline S.D. & 0.5 & 0.6 & 0.6 & 0.4 & 0.6 & 0.5 & 0.5 & 0.5 & 0.7 & 0.9 & 0.9 & 0.9 & 0.9 & 0.8 & 1.1 & 0.9 & 0.9 & 1.0 & 1.0 & 0.8 & 0.9 \\
\hline \multicolumn{22}{|c|}{ UPDRS } \\
\hline 1 & 9.0 & 8.0 & 10.0 & 9.0 & 10.0 & 9.0 & 9.2 & 8.0 & 5.0 & 4.0 & 4.0 & 4.0 & 4.0 & 4.8 & 5.0 & 8.0 & 9.0 & 10.0 & 10.0 & 10.0 & 8.7 \\
\hline 2 & 7.0 & 8.0 & 7.0 & 7.0 & 8.0 & 7.0 & 7.3 & 6.0 & 6.0 & 6.0 & 6.0 & 6.0 & 6.0 & 6.0 & 6.0 & 8.0 & 8.0 & 8.0 & 8.0 & 9.0 & 7.8 \\
\hline 3 & 8.0 & 7.0 & 7.0 & 8.0 & 8.0 & 8.0 & 7.7 & 7.0 & 6.0 & 6.0 & 6.0 & 6.0 & 6.0 & 6.2 & 6.0 & 8.0 & 7.0 & 8.0 & 8.0 & 8.0 & 7.5 \\
\hline 4 & 9.0 & 10.0 & 10.0 & 9.0 & 11.0 & 10.0 & 9.8 & 9.0 & 7.0 & 7.0 & 6.0 & 6.0 & 6.0 & 6.8 & 6.0 & 9.0 & 9.0 & 10.0 & 11.0 & 11.0 & 9.3 \\
\hline 5 & 8.0 & 7.0 & 8.0 & 8.0 & 8.0 & 8.0 & 7.8 & 6.0 & 5.0 & 4.0 & 4.0 & 4.0 & 4.0 & 4.5 & 6.0 & 8.0 & 8.0 & 7.0 & 8.0 & 9.0 & 7.7 \\
\hline 6 & 9.0 & 9.0 & 10.0 & 10.0 & 9.0 & 9.0 & 9.3 & 9.0 & 8.0 & 8.0 & 8.0 & 8.0 & 8.0 & 8.2 & 8.0 & 9.0 & 9.0 & 10.0 & 11.0 & 10.0 & 9.5 \\
\hline $7^{\mathrm{a}}$ & 9.0 & 8.0 & 8.0 & 9.0 & 9.0 & 9.0 & 8.7 & 7.0 & 5.0 & 5.5 & 4.5 & 4.5 & 5.0 & 4.8 & 6.0 & 8.0 & 8.0 & 9.0 & 8.0 & 9.0 & 8.0 \\
\hline 8 & 7.0 & 8.0 & 7.0 & 8.0 & 8.0 & 8.0 & 7.7 & 5.0 & 5.0 & 5.0 & 5.0 & 4.0 & 4.0 & 4.7 & 5.0 & 8.0 & 7.0 & 8.0 & 8.0 & 8.0 & 7.3 \\
\hline 9 & 8.0 & 7.0 & 8.0 & 7.0 & 8.0 & 8.0 & 7.7 & 6.0 & 5.0 & 4.5 & 5.0 & 4.5 & 4.5 & 4.5 & 6.0 & 8.0 & 8.0 & 7.0 & 8.0 & 9.0 & 7.7 \\
\hline $10^{\mathrm{a}}$ & 9.0 & 9.0 & 8.0 & 9.0 & 9.0 & 8.8 & 8.8 & 6.0 & 5.0 & 4.5 & 4.0 & 4.0 & 4.5 & 4.3 & 6.0 & 8.0 & 8.0 & 9.0 & 8.0 & 9.0 & 8.0 \\
\hline Mean & 8.3 & 8.1 & 8.3 & 8.4 & 8.8 & 8.5 & 8.4 & 6.9 & 5.7 & 5.3 & 5.0 & 5.0 & 5.0 & 5.5 & 6.0 & 8.2 & 8.1 & 8.6 & 8.8 & 9.2 & 8.2 \\
\hline S.D. & 0.8 & 1.0 & 1.3 & 1.0 & 1.0 & 0.8 & 1.0 & 1.4 & 1.1 & 1.4 & 1.5 & 1.4 & 1.4 & 1.4 & 0.8 & 0.4 & 0.7 & 1.2 & 1.3 & 0.9 & 0.9 \\
\hline
\end{tabular}

${ }^{\text {aP }}$ atients undergone an LD challenge after delivery of STN-DBS

Greenhause-Geisser correction). Post hoc Sheffé test showed the following: basal versus first LD hour $P=0.000034$; basal and first LD hour versus 2nd LD hour, NS. Friedman ANOVA followed by Wilcoxon test confirmed these significances. The average GABA decrease was $25 \%$ in the first hour and $15.9 \%$ in the second hour. LD effect was significant from the third fraction (from 20 to $30 \mathrm{~min}$ ) up to the third fraction of the second hour (Table 2 and Figure 3).

Pallidal cGMP changes. LD did not produce a significant change (Figure 3), although the mean cGMP concentration decreased from $1.89 \mathrm{fmol} / 50 \mu \mathrm{l}$ in the basal fractions to $1.65 \mathrm{fmol} / 50 \mu \mathrm{l}$ in the fractions from the first hour, and to $1.64 \mathrm{fmol} / 50 \mu \mathrm{l}$ during the second hour. Friedman ANOVA followed by Wilcoxon test confirmed this lack of significance. However, examination of the individual patients' data showed a trend for no change in subjects presenting with low cGMP basal values ( $<1.5 \mathrm{fmol}$ close to the detection limit), but a clear decrease (approximately 50\%) after LD administration in subjects presenting with higher basal values ( $>3.0 \mathrm{fmol}$, patient-2 and 6; highlighted in Table 2). This suggested a 'floor' effect in subjects with low basal values.

Clinical effect of $L D$. Friedman ANOVA showed a significant change of UPDRS score after LD administration $(n=6$; $\left.\mathrm{df}=17 ; \chi^{2}=83.13 ; P=0.000001\right)$. Post hoc Wilcoxon test showed a significant UPDRS score amelioration $30 \mathrm{~min}$ after LD administration in comparison with all the basal scores. The score was significantly lower up to $80 \mathrm{~min}$ after LD administration.

Correlations. A significant correlation was observed between GABA and cGMP changes (Pearson correlation $r=0.97$, $t=8.01, P=0.0013$ ), and between GABA and clinical score.

A closer look, case by case, at the GABA decrease and the UPDRS scores in both sub-groups (LD, alias melevodopa, and DBS; Figure 4) allows to recognize the homogeneity of the response following the drug challenge (Figure 4, left plot), 


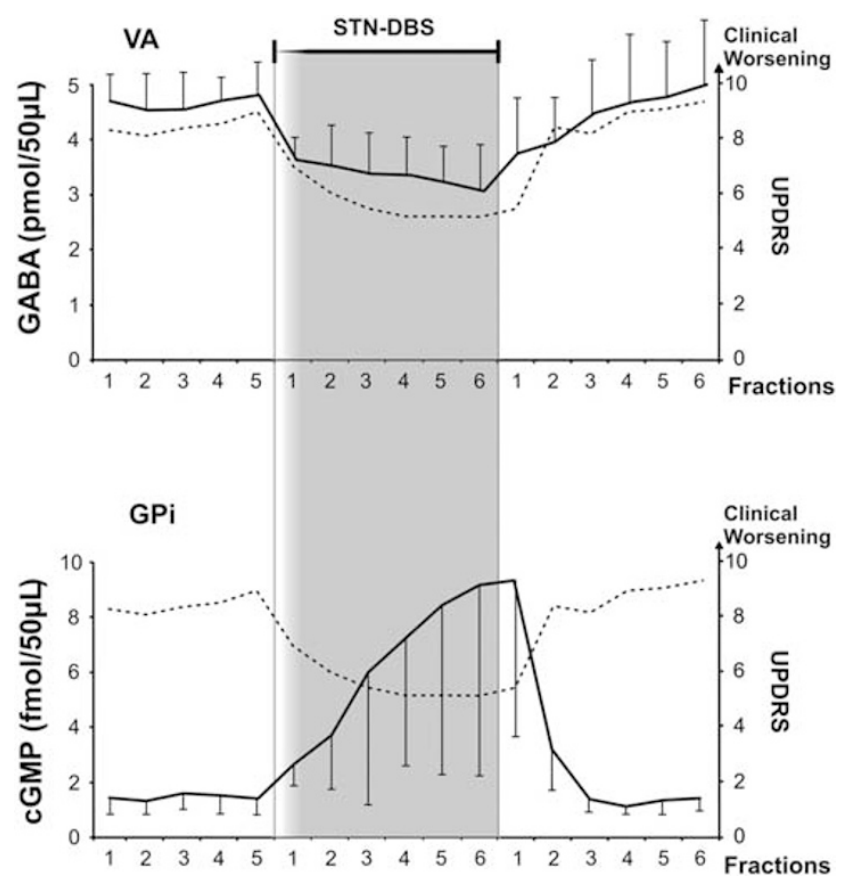

Figure 2 Microdialysis data from the GPi and VA, and clinical evaluation before, during, and after STN-DBS. Clinically effective STN-DBS caused a clear augmentation of cGMP in the GPi and a statistical significant GABA reduction in the VA (see section Results). The clinical scores at the bottom (dotted line, selected items 22, 24, and 25 from UPDRS III) validate the effectiveness of the STN-DBS. The data are expressed as the mean \pm S.D. whereas the DBS response features a much larger deviation (Figure 4, right plot). In many, but not all, patients the degree of clinical improvement tends to correlate with the change of GABA concentrations (see, for instance, cases 2-4 of the STIM group). Of course, the limited amount of cases discouraged further interpretations.

Finally, in two cases it was possible, given the patients' cooperation, to perform an LD challenge after the delivery of STN-DBS in the same surgery session (assuming that most of the DBS-mediated effects were over after an 80-min STIM cessation). Consider the example in Figure 5, which highlights the similar effects on GABA levels of $185-\mathrm{Hz}$ DBS and $100-\mathrm{mg}$ LD on the very same PD patient.

\section{Discussion}

GABA decrease in the VA thalamus, during effective STN-DBS or following LD administration, appears consistent with some aspects of the established model of BG function. ${ }^{1,2}$ Reduced GABA levels within the motor thalamus should dis-inhibit VA firing activity, with a profound impact on its patterning; reduced bursting and/or suppressed oscillatory activity are the most likely results, as inferred. ${ }^{22}$ The precise firing properties of the human VA/VL during effective STN-DBS are unknown, but previous studies in NHP may help the interpretation.

Extracellular recordings in MPTP-intoxicated primates documented, during STN-DBS, the relevant modification in the pattern of neuronal activity in the pallidal and cerebellar

Table 2 Microdialysis values and clinical scores before and during the first and second hour after LD intake

\begin{tabular}{|c|c|c|c|c|c|c|c|c|c|c|c|c|c|c|c|c|c|c|c|c|c|}
\hline \multirow[b]{3}{*}{ Patient } & \multicolumn{7}{|c|}{ Basal } & \multicolumn{7}{|c|}{ First hour after LD } & \multicolumn{7}{|c|}{ Second hour after LD } \\
\hline & \multicolumn{20}{|c|}{ cGMP-GPi } & \multirow[b]{2}{*}{ Mean } \\
\hline & & & & & & & Mean & & & & & & & Mean & & & & & & & \\
\hline 1 & 1.3 & 1.1 & 1.3 & 1.2 & 1.4 & 1.3 & 1.3 & 1.4 & 1.4 & 1.2 & 1.3 & 1.4 & 1.3 & 1.3 & 1.2 & 1.3 & 1.4 & 1.2 & 1.1 & 1.3 & 1.3 \\
\hline $2^{a}$ & 3.2 & 3.0 & 3.0 & 3.1 & 3.1 & 3.1 & 3.1 & 3.0 & 2.9 & 2.4 & 2.1 & 1.9 & 1.9 & 2.4 & 2.1 & 2.0 & 1.9 & 2.3 & 2.7 & 3.0 & 2.3 \\
\hline 3 & 1.3 & 1.3 & 1.7 & 1.1 & 1.5 & 1.4 & 1.4 & 1.3 & 1.5 & 1.5 & 1.3 & 1.5 & 1.6 & 1.5 & 1.4 & 1.3 & 1.3 & 1.7 & 1.2 & 1.3 & 1.4 \\
\hline 4 & 1.2 & 1.4 & 1.2 & 1.3 & 1.2 & 1.3 & 1.3 & 1.4 & 1.2 & 1.4 & 1.3 & 1.2 & 1.3 & 1.3 & 1.2 & 1.3 & 1.3 & 1.2 & 1.2 & 1.3 & 1.3 \\
\hline 5 & 1.5 & 1.2 & 1.3 & 1.3 & 1.2 & 1.3 & 1.3 & 1.4 & 1.2 & 1.3 & 1.2 & 1.2 & 1.3 & 1.3 & 1.3 & 1.1 & 1.3 & 1.4 & 1.4 & 1.5 & 1.3 \\
\hline $6^{a}$ & 3.0 & 3.1 & 3.2 & 3.1 & 3.0 & 3.1 & 3.1 & 2.9 & 3.0 & 2.4 & 2.1 & 1.4 & 1.3 & 2.2 & 1.9 & 2.0 & 2.1 & 2.3 & 2.7 & 3.0 & 2.3 \\
\hline Mean & 1.9 & 1.9 & 2.0 & 1.9 & 1.9 & 1.9 & 1.9 & 1.9 & 1.9 & 1.7 & 1.6 & 1.4 & 1.5 & 1.7 & 1.5 & 1.5 & 1.6 & 1.7 & 1.7 & 1.9 & 1.6 \\
\hline S.D. & 0.9 & 0.9 & 0.9 & 1.0 & 0.9 & 0.9 & 0.9 & 0.8 & 0.8 & 0.6 & 0.4 & 0.3 & 0.3 & 0.5 & 0.4 & 0.4 & 0.4 & 0.5 & 0.8 & 0.9 & 0.5 \\
\hline \multicolumn{22}{|c|}{ GABA-VA } \\
\hline 1 & 4.9 & 4.3 & 4.0 & 4.3 & 4.0 & 4.3 & 4.3 & 4.5 & 4.0 & 3.2 & 3.0 & 2.9 & 2.7 & 3.4 & 3.0 & 3.2 & 3.2 & 3.7 & 4.0 & 4.0 & 3.5 \\
\hline 2 & 4.1 & 4.3 & 4.9 & 4.3 & 4.5 & 4.4 & 4.4 & 4.2 & 4.0 & 3.2 & 3.0 & 2.5 & 2.3 & 3. & 2.4 & 2.3 & 3.0 & 3.5 & 4.0 & 4.0 & 3.2 \\
\hline 3 & 4.2 & 4.5 & 4.3 & 3.9 & 4.1 & 4.2 & 4.2 & 4.1 & 4.0 & 3.2 & 3.0 & 2.8 & 2.8 & 3.3 & 2.8 & 3.0 & 3.3 & 4.2 & 4.5 & 4.2 & 3.7 \\
\hline 4 & 4.2 & 4.3 & 4.3 & 4.7 & 4.3 & 4.4 & 4.4 & 4.2 & 4.1 & 3.0 & 3.0 & 3.1 & 2.7 & 3.4 & 2.9 & 3.3 & 4.0 & 4.0 & 4.2 & 4.5 & 3.8 \\
\hline 5 & 4.6 & 4.7 & 4.9 & 4.7 & 4.6 & 4.7 & 4.7 & 4.0 & 3.7 & 3.3 & 3.5 & 3.2 & 3.1 & 3.5 & 3.3 & 4.2 & 5.1 & 4.6 & 4.7 & 5.0 & 4.5 \\
\hline 6 & 4.9 & 4.5 & 4.2 & 4.3 & 4.3 & 4.4 & 4.4 & 4.2 & 4.0 & 3.0 & 3.2 & 2.2 & 2.0 & 3.1 & 2.3 & 2.3 & 3.2 & 3.6 & 4.1 & 4.2 & 3.3 \\
\hline Mean & 4.5 & 4.4 & 4.4 & 4.4 & 4.3 & 4.4 & 4.4 & 4.2 & 4.0 & 3.2 & 3.1 & 2.8 & 2.6 & 3.3 & 2.8 & 3.1 & 3.6 & 3.9 & 4.3 & 4.3 & 3.7 \\
\hline S.D. & 0.4 & 0.2 & 0.4 & 0.3 & 0.2 & 0.2 & 0.3 & 0.2 & 0.1 & 0.1 & 0.2 & 0.4 & 0.4 & 0.2 & 0.4 & 0.7 & 0.8 & 0.4 & 0.3 & 0.4 & 0.5 \\
\hline \multicolumn{22}{|l|}{ UPDRS } \\
\hline 1 & 10.0 & 8.0 & 8.0 & 10.0 & 9.0 & 9.0 & 9.0 & 10.0 & 8.0 & 6.0 & 5.0 & 5.0 & 5.0 & 6.5 & 5.0 & 5.0 & 7.0 & 8.0 & 10.0 & 9.0 & 7.3 \\
\hline 2 & 7.0 & 8.0 & 7.0 & 7.0 & 8.0 & 8.0 & 7.5 & 8.0 & 7.0 & 5.0 & 4.0 & 4.0 & 4.0 & 5.3 & 5.0 & 5.0 & 7.0 & 8.0 & 9.0 & 8.0 & 7.0 \\
\hline 3 & 7.0 & 8.0 & 8.0 & 7.0 & 8.0 & 8.0 & 7.7 & 8.0 & 8.0 & 5.0 & 5.0 & 4.0 & 4.0 & 5.7 & 4.0 & 5.0 & 5.0 & 8.0 & 8.0 & 8.0 & 6.3 \\
\hline 4 & 10.0 & 9.0 & 11.0 & 10.0 & 9.0 & 10.0 & 9.8 & 9.0 & 9.0 & 7.0 & 6.0 & 6.0 & 5.0 & 7.0 & 6.0 & 9.0 & 10.0 & 9.0 & 9.0 & 11.0 & 9.0 \\
\hline 5 & 8.0 & 8.0 & 8.0 & 8.0 & 8.0 & 8.0 & 8.0 & 8.0 & 8.0 & 6.0 & 6.0 & 5.0 & 5.0 & 6.3 & 5.0 & 7.0 & 10.0 & 8.0 & 8.0 & 8.0 & 7.7 \\
\hline 6 & 7.0 & 8.0 & 7.0 & 7.0 & 8.0 & 8.0 & 7.5 & 8.0 & 7.0 & 5.0 & 4.0 & 4.0 & 4.0 & 5.3 & 4.0 & 4.0 & 6.0 & 8.0 & 8.0 & 8.0 & 6.3 \\
\hline Mean & 8.2 & 8.2 & 8.2 & 8.2 & 8.3 & 8.5 & 8.3 & 8.5 & 7.8 & 5.7 & 5.0 & 4.7 & 4.5 & 6.0 & 4.8 & 5.8 & 7.5 & 8.2 & 8.7 & 8.7 & 7.3 \\
\hline S.D. & 1.5 & 0.4 & 1.5 & 1.5 & 0.5 & 0.8 & 1.0 & 0.8 & 0.8 & 0.8 & 0.9 & 0.8 & 0.5 & 0.8 & 0.8 & 1.8 & 2.1 & 0.4 & 0.8 & 1.2 & 1.2 \\
\hline
\end{tabular}

aPatients showing higher basal cGMP levels (see text) 

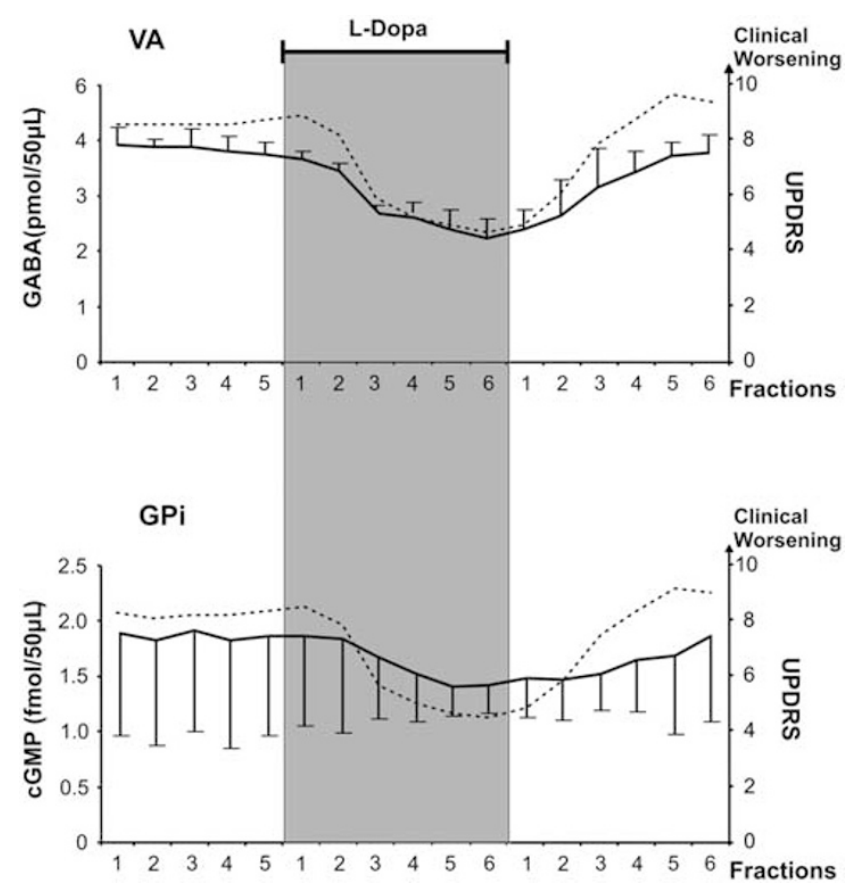

Figure 3 Microdialysis data from the GPi and VA, and clinical evaluation before and during LD (100 mg melevodopa) administration. In agreement with STN-DBS treatment, $L D$ cause a statistically significant GABA reduction in the VA (upper part). $L D$ produced a significant GABA decrease from the third fraction up to $50 \mathrm{~min}$. On the contrary, LD induced a negligible effect on GPi cGMP; a decrease of cGMP levels, if any, was observed in two patients (highlighted patients in Table 2, no. 2 and no. 6; see section Results). The data are expressed as the mean \pm S.D. Melevodopa (LD methyl ester) is a soluble form of LD, ${ }^{47}$ not currently in use in the United States, but routinely used in Italy (Sirio, by Chiesi Pharmaceuticals, Parma, Italy) in order to obtain a relatively fast $L D$-like response (plasma peak at $20-50 \mathrm{~min}$ )

\section{GABA and UPDRS \% decline}

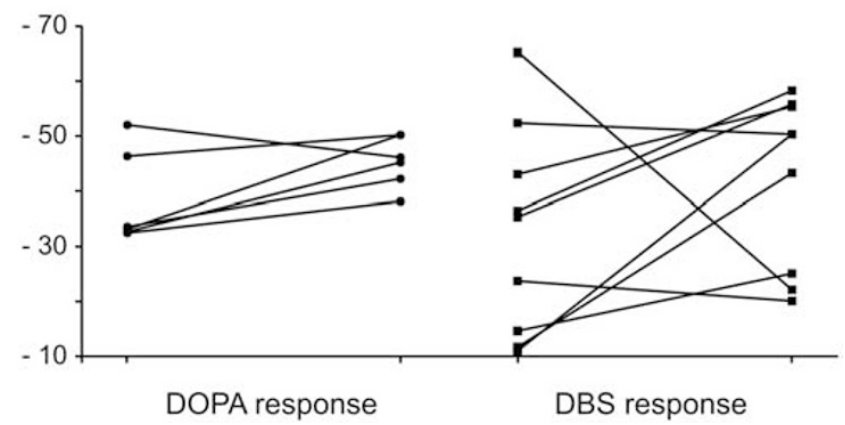

Figure 4 Correlation between biochemical change of VA GABA and clinica score. The plot shows in detail, case by case, the GABA and UPDRS decline during the drug response (left plot) and the DBS effect (right plot). The data are presented as \% decrease. The biochemical data are the average of the last two fractions before the start of each challenge. The clinical score represents the average of the last two fractions of the first hour following LD challenge or DBS switch-on. Note, on the left, the relative homogeneity of the response (comprised between 32 and $53 \%$ )

(VL) receiving areas of the motor thalamus. Both regions showed a more periodic and regular pattern, and a shift in oscillatory activity from low to high frequencies. ${ }^{14}$

Further, the Rubin and Terman ${ }^{12}$ model emphasized that a beneficial STN-DBS induces clustered firing patterns in GPi, whose clear consequence is a regularization of VA discharge (more tonic, no pausing). As pointed out by Guo et al.,

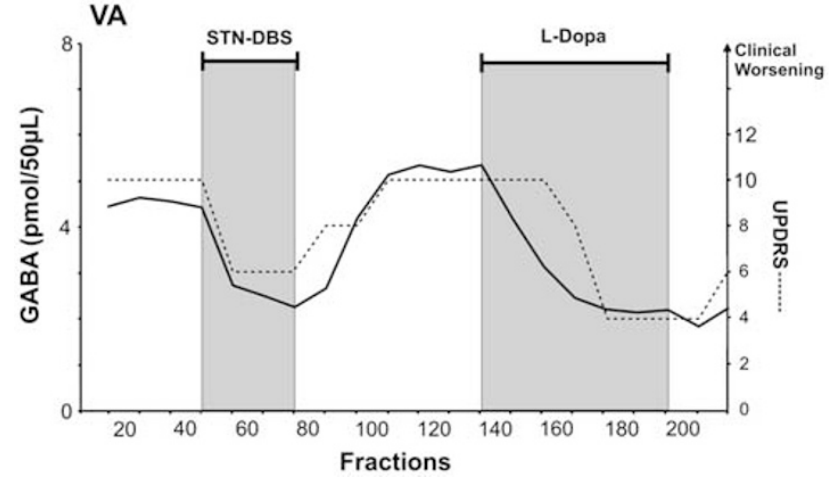

Figure 5 Microdialysis data showing changes of GABA in the VA and of clinical scores before and during STN-DBS and, subsequently, following LD ( $100 \mathrm{mg}$ melevodopa) administration. A similar reduction of GABA is produced by both treatments. The protocol was slightly shortened with respect to routine-1 (i.e. $30 \mathrm{~min}=3$ samples for DBS response) and was stopped before complete recovery of GABA levels in order to reduce surgery time

STN-DBS altered Parkinsonian GPi activity in a way that may improve thalamo-cortical relay fidelity. ${ }^{12,22}$ Accordingly, the reduced GABA levels that we observed would impede prolonged post-spiking after hyper-polarization, and favors a more regular pattern of neuronal activity.

As an additional confirmation of the clinical relevance of the reduced levels of GABA in the VA, consider the relationship between the degree of GABA decline under LD and the degree of LD-mediated clinical benefit (Figure 4). This is even more reliable when patients under evaluation are, as here, those in whom pharmacological sensitivity to LD was consistently assessed during the pre-surgery phase.

Thalamic VA/VL nuclei form a crucial link between BG outputs and specific cortical areas. ${ }^{23}$ It is reasonable to speculate that, once VA-VL is modulated by STN-DBS (as a consequence of reduced GABA release), immediate changes in thalamo-cortical transmission occur. PET studies reported a re-activation of fronto-temporo-parietal cortex at rest after effective STN-DBS (Hilker et al., ${ }^{24}$ but see Hershey et al.., ${ }^{25}$ ). Even when high-frequency STN-DBS decreased rCBF in the motor cortex at rest - implying a reduction of abnormal overactivity in the motor system - this allowed selective cortical reactivation during movement. ${ }^{26}$ Further, increased, movement-related, activity in $\mathrm{MC}$ followed efficacious pallidal $\mathrm{DBS}^{27}$ and STN-DBS in primates (J Vitek, unpublished).

It is difficult to match the GABA decrease in the VA with the increased cGMP reported in the GPi (or SNr) during STN-DBS (this paper and Galati et al. ${ }^{9}$, Stefani et al. ${ }^{20}$ ), indicative of increased GPi firing. As a matter of fact, the NOS/guanylate cyclase (sGC) pathway is mainly activated by glutamatergic excitatory transmission and extracellular cGMP, which is monitored by intra-cerebral microdialysis, reflects the in vivo functional state of this neurochemical pathway (for a review, see Pepicelli et al. ${ }^{28}$ ). Thus, the augmentation of cGMP in the $\mathrm{GPi}$ is a clear, although indirect, measure of pallidal neuron excitation driven by DBS-mediated glutamate release.

In principle, these findings do not fit with the early BG model, at least when suggesting that modulation of the hyper-excitability of the 'indirect pathways' is critical to rescue from akinesia. 
However, the cGMP data are not artifactual (and acquired from severe PD patients!); in addition, cGMP correlated with clinical benefits, whereas it was null if DBS was fake (0 V; Figure 1 in Stefani et al. ${ }^{20}$ ). Besides, distribution studies have shown that nNOS and soluble sGC (both $\alpha-1$ and $\beta-1$ subunits) are present at relatively high levels in the GPi of the human brain. ${ }^{29,30}$ These studies, although not determining a precise cellular localization indeed provide the biochemical basis for nitric oxide/cGMP production in this brain region.

Furthermore, the large DBS-mediated increase of GPi cGMP matches with an abundant literature. Strong support for an 'increased' GPi functional activity was raised by PET studies in PD patients, ${ }^{25}$ electrophysiological studies in MPTP-treated primates, ${ }^{8}$ increased cytochrome oxidase subunit-1 (COI) mRNA expression, ${ }^{31}$ and modeling studies supporting axonal activation of the STN neurons during STN-DBS. ${ }^{32}$

So, the issue is not whether an increased firing in the GPi/SNr during STN-DBS is observed (several demonstrations are available), rather to what extent this correlates with the decreased thalamic GABA.

One possibility is that the STN-DBS-mediated increase of GPi neuronal activity leads to the reduction of thalamic GABA release owing to the frequency-dependent activation of inhibitory $\mathrm{GABA}_{\mathrm{B}}$ auto-receptors on VA GABAergic terminals, as it happens in the hippocampus during the induction of long-term potentiation. ${ }^{33}$

In the anterior thalamus, complex synaptic arrangements, including triads of three GABAergic synapses (pallidal and reticular fibers with local inter-neurons or projection cell dendrites) and serial synapses with local GABAergic interneurons that in turn establish synaptic contacts with projection cell dendrites, compose up to $50 \%$ of synaptic contacts. ${ }^{34}$ Therefore, it could be hypothesized that DBS-driven GPi activity would enhance GABA release from GPi fibers onto VA-VL GABAergic inter-neurons, which in turn would decrease synaptic inhibition on thalamic glutamatergic neurons, finally re-activating the thalamo-cortical pathway. If this were the case, the small, though significant, decrease of thalamic GABA levels we observed could underestimate a more marked reduction in release from thalamic GABAergic inter-neurons.

Another possible explanation is that the electric field around the STN-DBS electrode inhibits large (1.5-5.0 $\mu \mathrm{m}$ diameter) GPi fibers directed to the thalamus and passing along the dorsal border of the STN, while simultaneously exciting thin STN-GPi/SNr fibers. ${ }^{35}$

Third, an augmentation of cGMP levels may reflect regularization of neuronal activity as well as an increase in the mean discharge rate, and it may be the regularization that is more important in modifying the GABA levels in the thalamus. As noted previously, STN-DBS act as a modulator of endogenous oscillations. ${ }^{22}$ One model predicts that even during an (apparent) increase of GPi discharge, the more tonic firing pattern may impede irregular GABA release (propelled by spike trains) on target thalamo-cortical cells, restoring their responsiveness to sensory inputs. ${ }^{12}$

Alternatively, the two examined effects of DBS (increased cGMP in GPi and lower GABA in VA) might not be necessarily linked. Actually, STN-DBS reverberates on multiple sites, including a ventral tegmental area and pedunculopontine nucleus extended area, ${ }^{36}$ which in turn affects the ascending pathways toward the thalamus. In other words, the proteiform response to DBS on distant structures might overcome the strict boundaries of the direct/indirect pathway scheme.

In line with DBS-mediated mechanisms, LD promoted a similar decrease of GABA levels in the VA, together with a significant clinical improvement. To note, the LD-mediated decline of GABA was clearly more homogeneous than the DBS response (see Figure 4). In contrast with DBS, the LD-induced decline of GABA in the VA coincides with a reduction of GPi activity, ${ }^{3,37-39}$ but with a negligible effect on GPi cGMP levels. In our experience, the apomorphine response in the GPi of human PD patients was characterized by a dose-dependent fast inhibition and showed partial desensitization. ${ }^{40}$

However, two subjects with higher basal levels of cGMP showed a clear decrease after LD administration, suggesting that LD might reduce GPi cGMP, an effect hard to detect if basal cGMP levels are close to the detection limit (as in the other four patients).

On the other hand, lack of cGMP change during LD challenge may imply functional de-rearrangements of the dopamine-cGMP interplay in advanced PD patients.

It has been reported that unilateral lesions of the nigrostriatal pathway with 6-OHDA in rats resulted in a significant decrease of cGMP levels in the ipsilateral caudate-putamen (CP) and GP. ${ }^{41,42}$ Interestingly, in 6-OHDA-lesioned eukinetic rats administration of LD failed to affect the cGMP levels in the ipsilateral CP and GP, whereas it was able to decrease those levels in the same regions on the non-lesioned contralateral side; on the contrary, LD lowered cGMP levels in both hemispheres in 6-OHDA-lesioned rats at the peak of LDinduced dyskinesia. ${ }^{42}$ These results highlight that the cGMP response to $L D$ in the $B G$ could be different under dyskinetic and non-diskynetic conditions, and might explain the lack of LD-induced changes of cGMP in the GPi, as LD was administered to patients at a sub-dyskinetic dose.

Functional implications. The core finding of this investigation is the consistent and clinically related GABA decline in the motor thalamus, which may associate (during DBS) or may not associate (under LD) with simultaneous changes of GPi cGMP. Hence, the clinically relevant modulation of GABA in the VA occurs despite or besides ongoing changes of pallidal discharge. Parsimonious implications are that, during LD-mediated clinical amelioration, pathways other than the pallido-thalamic, but still affecting the VA, indeed contribute to the rebalancing of the thalamo-cortical output.

That said, the common effect on VA GABA levels, shared by both treatments, indicates that this reduction reflects some degree of mechanistic similarity underlying the clinical effects of STN-DBS and LD.

Few centers still use unilateral pallidotomy as a competitive surgical approach ${ }^{43}$ (of course, in advanced PD patients affected by disabling dyskinesias and reluctant to undergo bilateral DBS). Pallidotomy would suppress ipsilateral GABA transmission in the VA/VL. Our present data lend support to a 
putative re-evaluation of selective ablations (to interfere with endogenous release of GABA in VA) or, speculatively, to the implementation of devices aimed at rebalancing intra-thalamic GABA levels.

The dichotomy between the STN- and LD-mediated effects on the cGMP levels in GPi is not, per se, surprising if we take into account their different electrophysiological effects. In contrast with DBS, which may excite and/or cluster neuronal activity, exogenous LD has a strong inhibitory influence on the neuronal activity of $\mathrm{GPi} .{ }^{38}$ An almost complete suppression of GPi discharge associates with LD-induced dyskinesia! $!^{38,44}$

In conclusion, a number of investigations from multiple disciplines support the hypothesis that stimulation-induced modulation of pathological network activity represents the most likely mechanism of DBS. ${ }^{45}$ Recent contribution (by using an optogenic approach) suggested that highfrequency STN stimulation, in 6-OHDA rodents, promotes mostly a direct activation of cortical axons to STN. ${ }^{46}$

Nevertheless, in agreement with recent conclusions of Johnson et al. ${ }^{27}$ ('DBS improves Parkinsonian motor symptoms by inducing global changes in firing pattern and rate along the pallido-thalamocortical sensorimotor circuit'), our results further emphasize the motor thalamus as the critical station where efficacious re-modulation (electrical or pharmacological) of pathological outputs promotes good therapeutic outcomes.

\section{Materials and Methods}

Patients. Sixteen advanced PD patients (mean age $=58.9 \pm 6.5$ years; mean UPDRS score in OFF $=46.7 \pm 9.1$; mean disease duration $=11.4 \pm 3.7$ years) were included in the study according to previously reported selection criteria. ${ }^{19,38,39}$ For epidemiological data, see Table 3. Response to STN-DBS was analyzed in 10 patients and response to L-DOPA was monitored in six patients. Actually, we routinely used melevodopa (Sirio $100+25$ ), a soluble analog of LD/carbidopa introduced since early 2000s in clinical practice in Italy. Given its pharmacokinetic profile, ${ }^{47}$ melevodopa allows to obtain a consistent peak response in 20-50 min, still

Table 3 Clinical characteristics of the 16 PD patients in the study

\begin{tabular}{|c|c|c|c|c|}
\hline PD patient & $\begin{array}{c}\text { Age } \\
\text { (years) }\end{array}$ & $\begin{array}{c}\text { Disease } \\
\text { duration } \\
\text { (years) }\end{array}$ & $\begin{array}{c}\text { L-DOPA } \\
\text { duration } \\
\text { (years) }\end{array}$ & $\begin{array}{c}\text { L-DOPA } \\
\text { daily } \\
\text { dose (mg) }\end{array}$ \\
\hline \multicolumn{5}{|l|}{$D B S$} \\
\hline 1 & 68 & 10 & 10 & 750 \\
\hline 2 & 60 & 23 & 23 & 800 \\
\hline 3 & 52 & 10 & 7 & 600 \\
\hline 4 & 49 & 15 & 13 & 900 \\
\hline 5 & 59 & 12 & 12 & 1000 \\
\hline 6 & 56 & 13 & 12 & 800 \\
\hline 7 & 63 & 10 & 10 & 1000 \\
\hline 8 & 64 & 11 & 11 & 1300 \\
\hline \multicolumn{5}{|l|}{$D B S+L-D O P A$} \\
\hline 9 & 54 & 7 & 7 & 800 \\
\hline 10 & 65 & 9 & 9 & 1100 \\
\hline \multicolumn{5}{|l|}{ L-DOPA } \\
\hline 11 & 48 & 12 & 11 & 750 \\
\hline 12 & 54 & 11 & 6 & 1200 \\
\hline 13 & 62 & 10 & 8 & 750 \\
\hline 14 & 69 & 10 & 9 & 1050 \\
\hline 15 & 55 & 7 & 7 & 800 \\
\hline 16 & 65 & 13 & 9 & 1000 \\
\hline 17 & 58 & 9 & 7 & 750 \\
\hline 18 & 60 & 12 & 8 & 1325 \\
\hline Mean $( \pm)$ S.D. & $58.9 \pm 6.1$ & $11.3 \pm 3.5$ & $9.9 \pm 3.8$ & $925 \pm 210$ \\
\hline
\end{tabular}

maintaining an adequate steady-state response, at least in the $2 \mathrm{~h}$ examined here. In order to avoid misunderstanding, LD is used throughout the entire paper.

The two groups did not manifest significant differences in terms of disease history, duration, or LD dosage.

In addition, two PD patients accepted to undergo, in sequence, both STN-DBS and LD challenge, albeit with a shortened protocol.

Surgical and microdialysis technique. Permanent electrodes were implanted in both the STN and the GPi, bilaterally, according to our previous multi-target strategy ${ }^{48}$ For trajectory details, and implantation procedures, see our previous works ${ }^{19-21}$ and the legend in Figure 1.

Written, informed consent was obtained from each patient. The Local Ethics Committee of the University of Tor Vergata approved the protocol and the consent form describing the risks and potential benefits of the study. The protocol included the following step procedures: electrophysiological single-unit micro-recordings in STN for proper identification of the best trajectory for lead implantation; insertion of the permanent DBS lead (Medtronic 3389) into the STN (final coordinates= AC-PC/2, 11-12 mm lateral to the midline, and $4-5 \mathrm{~mm}$ below the AC-PC); insertion of guide tubes into the VA and GPi for microdialysis (only at the end of the microdialysis session the permanent electrode was positioned also in the GPi, whose coordinates are as follows (for the lowest contact): $2-3 \mathrm{~mm}$ anterior to the AC-PC/2, 19-22 $\mathrm{mm}$ lateral to the midline of the third ventricle, and $6 \mathrm{~mm}$ below the inter-commissural line: ${ }^{16}$ ). To note, microdialysis onto human Parkinsonian brain was pioneered by Ungerstedt, ${ }^{49}$ extensively used by our group, ${ }^{9,19-21}$ and recently replicated by Kilpatrick et al. ${ }^{50}$

As previously reported, ${ }^{19}$ the microdialysis concentric probes had the following features: external membrane diameter $0.6 \mathrm{~mm}$, external tube diameter $0.5 \mathrm{~mm}$, membrane PES with 20-kDa molecular weight cutoff, 4-mm dialyzing zone for GPi and VA (Metalant AB, Metalant, Sweden). The microdialysis probes were connected to a CMA100 microinjection pump (CMA Microdialysis, Stockholm, Sweden) set at $5 \mu \mathrm{l} / \mathrm{min}$. Perfusion was started immediately with a sterile PBS solution containing (in g/l) $\mathrm{NaCl}(8), \mathrm{KCl}(0.2), \mathrm{CaCl}_{2} \times 2 \mathrm{H}_{2} \mathrm{O}(0.132), \mathrm{MgCl}_{2} \times 6 \mathrm{H}_{2} \mathrm{O}(0.1), \mathrm{Na}_{2} \mathrm{HPO}_{4}$ (1.15), $\mathrm{KH}_{2} \mathrm{PO}_{4}(0.2)(\mathrm{pH} 7.4)$. The outlet of the probes was placed into plastic vials situated in a stand fitted to a stereotaxic frame. Complete sterility of all the materials used (probes connectors, tubing, vials, and solutions) was assured. Samples were collected every $10 \mathrm{~min}$ and immediately frozen. The amount of GABA in each fraction was determined by HPLC analysis coupled to fluorimetric detection. The buffers and gradient program used were those reported by Fedele and Raiteri. ${ }^{51}$ Homoserine was used as internal standard; the detection limit for GABA was approximately $100 \mathrm{fmol} / 40 \mu \mathrm{l}$. In order to ascertain that all the probes used sampled GABA with the same efficiency, at the end of the operation they were tested in vitro using standard solutions to determine the relative recovery of GABA (no significant difference for in vitro recovery has been observed among the probes used for different patients). The mean basal values of the amino acid collected by each probe in any patient were calculated from the tenth fractions (all fractions before assumed as under stabilization and, thus, discarded). cGMP concentration was determined by radio-immunoassay (RIA) as already described. ${ }^{20}$

At the end of the stabilization phase $(90 \mathrm{~min})$, basal microdialysis samples $(60 \mu \mathrm{l}$, $10 \mathrm{~min}$ each) and clinical data were collected for $60 \mathrm{~min}$ (see Figures 2 and 3 ). Afterwards, either STN-DBS was switched on for $60 \mathrm{~min}$ ( $n=10$ patients; Table 1 and Figure 2; the stimulation parameters for bipolar STIM were approximately $185 \mathrm{~Hz}$, mean $2 \mathrm{~V}$ (range 1.8-2.4), $60 \mu$ s duration etc.) or melevodopa was administered orally ( $100 \mathrm{mg}, n=6$ patients; Table 1 and Figure 3 ).

After a 60 -min DBS, $60 \mathrm{~min}$ of recovery were performed (Table 1). After LD administration, dialysis continued for further $120 \mathrm{~min}$ (two 60-min periods; Table 1).

All procedures were without additional risks for the patients, as the guide tubes for micro-recordings were already placed in each target (STN and GPi). That said, the microdialysis procedure per se indeed prolongs (an average of $3.5 \mathrm{~h}$ ) the entire surgery section. This is why only in special occasions it was possible to obtain microdialysis data under both clinical challenges, albeit with a shortened protocol (DBS followed by LD, $n=2$; see Figure 5), or it was possible to check the lack of GABA title changes during a 0-V STN-DBS ( $n=2$, not shown).

Clinical changes were assessed using a subset of the items of UPDRS (rigidity $0-4$, finger tapping $0-4$, hand movement $0-4$; total $=0$ corresponds to 'normal', 12 maximum score) by a neurologist blinded to the stimulus intensity between 0 and $3 \mathrm{~V}$ or the time of $L D$ administration.

Statistical analysis. Mean GABA and cGMP levels (expressed as pmol or fmol/50 $\mu$ l, respectively) were compared by two-way ANOVA (first main factor: 
treatment with three levels: basal, treatment (DBS, LD, or none), recovery; second main factor: time with six levels) corrected by Greenhause-Gaisser correction and followed by post hoc Sheffè test to assess the significance of the differences between single fractions. A similar procedure was used for $L D$ administration except the three levels of the first main factor were basal, LD1 (0-60 min after LD administration), and LD2 (60-120 min).

Given the possible non-normality of biochemical data, the results were also confirmed by non-parametric Friedman ANOVA followed by Wilcoxon post-hoc test. The clinical scores were compared using similar non-parametric methods. Correlations were studied between the maximal changes in the GABA and cGMP concentrations induced by DBS and LD (Pearson parametric correlations), and clinical score (non-parametric Spearman correlations). The changes were evaluated as the maximum change of each of these parameters in comparison with their mean basal score.

\section{Conflict of Interest}

The authors declare no conflict of interest.

Acknowledgements. This work has been supported by Ministero della Salute grants to PS and AS.

1. Albin RL, Young AB, Penney JB. The functional anatomy of basal ganglia disorders. Trends Neurosci 1989; 12: 366-375.

2. DeLong MR. Primate models of movement disorders of basal ganglia origin. Trends Neurosci 1990; 13: 281-285

3. Filali M, Hutchison WD, Palter VN, Lozano AM, Dostrovsky JO. Stimulation-induced inhibition of neuronal firing in human subthalamic nucleus. Exp Brain Res 2004; 156 274-281.

4. Benazzouz A, Gao DM, Ni ZG, Piallat B, Bouali-Benazzouz R, Benabid AL. Effect of highfrequency stimulation of the subthalamic nucleus on the neuronal activities of the substantia nigra pars reticulata and ventrolateral nucleus of the thalamus in the rat. Neuroscience 2000; 99: 289-295.

5. Windels F, Bruet N, Poupard A, Feuerstein C, Bertrand A, Savasta M. Influence of the frequency parameter on extracellular glutamate and gamma-aminobutyric acid in substantia nigra and globus pallidus during electrical stimulation of subthalamic nucleus in rats. J Neurosci Res 2003; 72: 259-267.

6. Windels F, Carcenac C, Poupard A, Savasta M. Pallidal origin of GABA release within the substantia nigra pars reticulata during high-frequency stimulation of the subthalamic nucleus. J Neurosci 2005; 25: 5079-5086.

7. Shi LH, Luo F, Woodward DJ, Chang JY. Basal ganglia neural responses during behaviorally effective deep brain stimulation of the subthalamic nucleus in rats performing a treadmill locomotion test. Synapse 2006; 59: 445-457.

8. Hashimoto T, Elder CM, Okun MS, Patrick SK, Vitek JL. Stimulation of the subthalamic nucleus changes the firing pattern of pallidal neurons. J Neurosci 2003; 23: 1916-1923.

9. Galati S, Mazzone P, Fedele E, Pisani A, Peppe A, Pierantozzi M et al. Biochemical and electrophysiological changes of substantia nigra pars reticulata driven by subthalamic stimulation in patients with Parkinson's disease. Eur J Neurosci 2006; 23: 2923-2928.

10. Hahn PJ, Russo GS, Hashimoto T, Miocinovic S, Xu W, Mclntyre CC et al. Pallidal burst activity during therapeutic deep brain stimulation. Exp Neurol 2008; 211: 243-251.

11. Maltête D, Jodoin N, Karachi C, Houeto JL, Navarro S, Cornu P et al. Subthalamic stimulation and neuronal activity in the substantia nigra in Parkinson's disease. J Neurophysiol 2007; 97: 4017-4022.

12. Rubin JE, Terman D. High frequency stimulation of the subthalamic nucleus eliminates pathological thalamic rhythmicity in a computational model. J Comput Neurosci 2004; 16 : 211-235.

13. Hassler R. Anatomy of the thalamus In: Schaltenbrand G, Baily P (eds) Introduction to Stereotaxis with an Atlas of the Human Brain. Stuttgart: Thieme, 1959, pp 230-290

14. Xu W, Russo GS, Hashimoto T, Zhang J, Vitek JL. Subthalamic nucleus stimulation modulates thalamic neuronal activity. J Neurosci 2008; 28: 11916-11924.

15. Vingerhoets FJ, Villemure JG, Temperli P, Pollo C, Pralong E, Ghika J. Subthalamic DBS replaces levodopa in Parkinson's disease: two-year follow-up. Neurology 2002; 58 : 396-401.

16. Peppe A, Pierantozzi M, Bassi A, Altibrandi MG, Brusa L, Stefani A et al. Stimulation of the subthalamic nucleus compared with the globus pallidus internus in patients with Parkinson disease. J Neurosurg 2004; 101: 195-200.

17. Pagonabarraga J, García-Sánchez C, Llebaria G, Pascual-Sedano B, Gironell A Kulisevsky J. Controlled study of decision-making and cognitive impairment in Parkinson's disease. Mov Disord 2007; 22: 1430-1435.

18. Nieoullon A. Dopamine and the regulation of cognition and attention. Prog Neurobiol 2002 67: 53-83.
19. Fedele E, Mazzone P, Stefani A, Bassi A, Ansaldo MA, Raiteri M et al. Microdialysis in Parkinsonian patient basal ganglia: acute apomorphine-induced clinical and electrophysiological effects not paralleled by changes in the release of neuroactive amino acids. Exp Neurol 2001; 167: 356-365.

20. Stefani A, Fedele E, Galati S, Pepicelli O, Frasca S, Pierantozzi M et al. Subthalamic stimulation activates internal pallidus: evidence from cGMP microdialysis in PD patients. Ann Neurol 2005; 57: 448-452.

21. Stefani A, Fedele E, Galati S, Raiteri M, Pepicelli O, Brusa L et al. Deep brain stimulation in Parkinson's disease patients: biochemical evidence. J Neural Transm 2006; 70 : S401-S408.

22. Guo Y, Rubin JE, McIntyre CC, Vietk JL, Terman D. Thalamocortical relay fidelity varies across subthalamic nucleus deep brain stimulation protocols in a data-driven computational model. J Neurophysiol 2008; 99: 1477-1492.

23. Obeso JA, Rodríguez-Oroz MC, Benitez-Temino B, Blesa FJ, Guridi J, Marin C et al. Functional organization of the basal ganglia: therapeutic implications for Parkinson's disease. Mov Disord 2008; 23: S548-S559.

24. Hilker R, Voges J, Weisenbach S, Kalbe E, Burghaus L, Ghaemi M et al. Subthalamic nucleus stimulation restores glucose metabolism in associative and limbic cortices and in cerebellum: evidence from a FDG-PET study in advanced Parkinson's disease. Cereb Blood Flow Metab 2004; 24: 7-16.

25. Hershey T, Revilla FJ, Wernle AR, McGee-Minnich L, Antenor JV, Videen TO et al. Cortical and subcortical blood flow effects of subthalamic nucleus stimulation in PD. Neurology 2003; 61: 816-821.

26. Payoux P, Remy P, Damier P, Miloudi M, Loubinoux I, Pidoux B et al. Subthalamic nucleus stimulation reduces abnormal motor cortical overactivity in Parkinson disease. Arch Neurol 2004; 61: 1307-1313.

27. Johnson MD, Vitek JL, McIntyre CC. Pallidal stimulation that improves parkinsonian motor symptoms also modulates neuronal firing patterns in primary motor cortex in the MPTP-treated monkey. Exp Neurol 2009; 219: 359-362.

28. Pepicelli O, Raiteri M, Fedele E. The NOS/sGC pathway in the rat central nervous system: a microdialysis overview. Neurochem Int 2004; 45: 787-797.

29. Blum-Degen D, Heinemann $T$, Lan J, Pedersen V, Leblhuber F, Paulus $W$ et al. Characterization and regional distribution of nitric oxide synthase in the human brain during normal ageing. Brain Res 1999; 834: 128-135.

30. Ibarra C, Nedvestsky PI, Gerlach M, Riederer P, Schmidt HHHW. Regional and agedependent expression of the nitric oxide receptor, soluble guanylyl cyclase, in the human brain. Brain Res 2001; 907: 54-60

31. Mitchell IJ, Clarke CE, Boyce S, Robertson RG, Peggs D, Sambrook MA et al. Neural mechanisms underlying parkinsonian symptoms based upon regional uptake of 2-deoxyglucose in monkeys exposed to 1-methyl-4-phenyl-1,2,3,6-tetrahydropyridine. Neuroscience 1989; 32: 213-226.

32. Miocinovic S, Parent M, Butson CR, Hahn PJ, Russo GS, Vitek JL et al. Computational analysis of subthalamic nucleus and lenticular fasciculus activation during therapeutic deep brain stimulation. J Neurophysiol 2006; 96: 1569-1580.

33. Davies CH, Starkey SJ, Pozza MF, Collingridge GL. GABA autoreceptors regulate the induction of LTP. Nature 1991; 349: 609-611.

34. Sidibé M, Bevan MD, Bolam JP, Smith Y. Efferent connection of the internal globus pallidus in the squirrel monkey: I topography and synaptic organization of the pallidothalamic projection. J Comp Neurol 1997; 382: 323-347.

35. McIntyre CC, Savasta M, Kerkerian-Le Goff L, Vitek JL. Uncovering the mechanism(s) of action of deep brain stimulation: activation, inhibition, or both. Clin Neurophysiol 2004; 115 1239-1248.

36. Stefani A, Lozano AM, Peppe A, Stanzione P, Galati S, Tropepi D et al. Bilateral deep brain stimulation of the pedunculopontine and subthalamic nuclei in severe Parkinson's disease. Brain 2007; 130: 1596-1607.

37. Hutchinson WD, Levy R, Dostrovsky JO, Lozano AM, Lang AE. Effects of apomorphine on globus pallidus neurons in parkinsonian patients. Ann Neurol 1997; 42 $767-775$.

38. Stefani A, Stanzione P, Bassi A, Mazzone P, Vangelista $T$, Bernardi G. Effects of increasing doses of apomorphine during stereotaxic neurosurgery in Parkinson's disease: clinical score and internal globus pallidus activity. J Neural Transm 1997; 104 895-904.

39. Stefani A, Bassi A, Mazzone P, Pierantozzi M, Gattoni G, Altibrandi MG et al. Subdyskinetic apomorphine responses in globus pallidus and subthalamus of parkinsonian patients: lack of clear evidence for the 'indirect pathway'. Clin Neurophysiol 2002; 113: 91-100

40. Stefani A, Mazzone P, Bassi A, Bernardi G, Altibrandi MG, Peppe A et al. Electrophysiological and clinical desensitization to apomorphine administration in parkinsonian patients undergoing stereotaxic neurosurgery. Exp Neurol 1999; 156 209-213.

41. Sancesario G, Giorgi M, D'Angelo V, Modica A, Martorana A, Morello M et al. Downregulation of nitrergic transmission in the rat striatum after chronic nigrostriatal deafferentation. Eur J Neurosci 2004; 20: 989-1000.

42. Giorgi M, D'Angelo V, Esposito Z, Nuccetelli V, Sorge R, Martorana A et al. Lowered CAMP and CGMP signalling in the brain during levodopa-induced dyskinesias in hemiparkinsonian rats: new aspects in the pathogenetic mechanisms. Eur $J$ Neurosc 2008; 28: 941-950 
43. Gross RE. What happened to posteroventral pallidotomy for Parkinson's disease and dystonia? Neurotherapeutics 2008; 5: 281-293.

44. Lozano AM, Lang AE, Levy R, Hutchison W, Dostrowsky J. Neuronal recordings in Parkinson's disease patients with dyskinesias induced by apomorphine. Ann Neurol 2000; 47: S141-S146

45. Brown P. Oscillatory nature of human basal ganglia activity: relationship to the pathophysiology of Parkinson's disease. Mov Disord 2003; 18: 357-363.

46. Gradinaru V, Mogri M, Thompson KR, Henderson JM, Deisseroth K. Optical deconstruction of parkinsonian neural circuitry. Science 2009; 324: 354-359.

47. Stocchi F, Quinn NP, Barbato L, Patsalos PN, O'Connel MT, Ruggieri S et al. Comparison between a fast and a slow release preparation of levodopa and a combination of the two: a clinical and pharmacokinetic study. Clin Neuropharmacol 1994; 17: 38-44.

48. Stefani A, Peppe A, Pierantozzi M, Galati S, Moschella V, Stanzione P et al. Multi-target strategy for Parkinsonian patients: the role of deep brain stimulation in the centromedianparafascicularis complex. Brain Res Bull 2009; 78: 113-118.
49. Meyerson BA, Linderoth $\mathrm{B}$, Karlsson $\mathrm{H}$, Ungerstedt $\mathrm{U}$. Microdialysis in the human brain: extracellular measurements in the thalamus of parkinsonian patients. Life Sci 1990; 46: 301-308.

50. Kilpatrick M, Church E, Danish S, Stiefel M, Jaggi J, Halpern C et al. Intracerebral microdialysis during deep brain stimulation surgery. J Neurosci Meth 2010; 190: 106-111.

51. Fedele E, Raiteri M. In vivo studies of the cerebral glutamate receptor/NO/cGMP pathway. Prog Neurobiol 1999; 58: 89-120.

c)

Cell Death and Disease is an open-access journal published by Nature Publishing Group. This work is licensed under the Creative Commons Attribution-Noncommercial-No Derivative Works 3.0 Unported License. To view a copy of this license, visit http://creativecommons.org/licenses/by-nc-nd/3.0/ 